\title{
Statistics of the sodium layer parameters at low geographic latitude and its impact on adaptive-optics sodium laser guide star characteristics
}

\author{
N. Moussaoui ${ }^{1,2}$, B. R. Clemesha ${ }^{3}$, R. Holzlöhner ${ }^{1}$, D. M. Simonich ${ }^{3}$, D. Bonaccini Calia ${ }^{1}$, \\ W. Hackenberg ${ }^{1}$, and P. P. Batista ${ }^{3}$ \\ ${ }^{1}$ European Organization for Astronomical Research in the Southern Hemisphere (ESO), Karl-Schwarzschild-Stra $\beta$ e 2, \\ 85748 Garching bei München, Germany \\ e-mail: nmoussao@eso.org \\ 2 Faculty of Physics, University of Sciences and Technology Houari Boumediene, BP32 El-Alia, Bab-Ezzouar, Algiers, Algeria \\ 3 Instituto National de Pesquisas Espaciais-MCT, São José dos Compos, São Paulo, Brazil
}

Received 11 September 2009 / Accepted 6 November 2009

\begin{abstract}
Aims. To aid the design of laser guide star (LGS) assisted adaptive optics (AO) systems, we present an analysis of the statistics of the mesospheric sodium layer based on long-term observations (35 years).

Methods. We analyze measurements of the Na-layer characteristics covering a long period (1973-2008), acquired at latitude $23^{\circ}$ south, in São José dos Compos, São Paulo, Brazil. We note that Paranal (Chile) is located at latitude $24^{\circ}$ south, approximately the same latitude as São Paulo.

Results. This study allowed us to assess the availability of LGS-assisted AO systems depending on the sodium layer properties. We also present an analysis of the LGSs spot elongation over the year, as well as the nocturnal and the seasonal variation in the mesospheric sodium layer parameters.

Conclusions. The average values of the sodium layer parameters are $92.09 \mathrm{~km}$ for the centroid height, $11.37 \mathrm{~km}$ for the layer thickness, and $5 \times 10^{13} \mathrm{~m}^{-2}$ for the column abundance. Assuming a laser of sufficient power to produce an adequate photon return flux for an AO system with a column abundance of $4 \times 10^{13} \mathrm{~m}^{-2}$, a telescope could observe at low geographic latitudes with the sodium LGS more than 250 days per year. Increasing this power by $20 \%$, we could observe throughout the entire year.
\end{abstract}

Key words. instrumentation: adaptive optics - atmospheric effects

\section{Introduction}

The mesospheric sodium layer exists between altitudes of 80 and $105 \mathrm{~km}$. Meteoric ablation is currently believed to be the source of the mesospheric sodium and the other metal layers in this region of the atmosphere (Plane 2003). The existence of this layer has been known since the middle of the 1930's. Long-term observations of the Na layer provided more details of seasonal, latitudinal and diurnal variations (Megie \& Blamont 1977; Simonich et al. 1978; Clemesha et al. 1979; Kirchhoff \& Clemesha 1983; Gardner et al. 1988; Tilgner \& von Zahn 1988; von Zahn et al. 1988; Plane et al. 1999; She et al. 2000; Clemesha et al. 2004; and Gardner et al. 2005).

Adaptive optics systems require a reference source of light for measuring and correcting wave-front distortions. This reference source of light must be in the field of view of the astronomical subject of interest and as close as possible to its line of sight. It is often not possible to find a natural source of light bright enough and close enough to each astronomical source of interest. For this reason, full sky coverage using adaptive optics is achieved by a laser beacon producing a laser guide star (LGS), which acts as a reference source to provide the required information about the turbulence-induced wavefront distortion produced below the beacon's altitude. Hence, the higher the LGS, the better is the information about the wavefront distortion. A LGS can be created by backscattered light from a ground-based laser beam pointed toward the scientific source and created by exciting the mesospheric sodium atoms using a laser beam tuned to the sodium $D_{2}$ line at $589 \mathrm{~nm}$. The characteristics of the LGS depend not only on the laser system itself but also on the properties of the mesospheric sodium layer. As adaptive optics systems of the first generation become mature technology, the accurate system design of the next generation of LGS-AO systems requires a detailed knowledge of the statistical properties of the mesospheric sodium layer. By using more than 30 years of Lidar observations produced by some of the authors, statistical properties of the mesospheric sodium layer at latitudes almost identical to the ESO Paranal observatory can be derived. The data extracted are those most needed for the LGS-AO system design of European Extremely Large Telescopes (E-ELT), such as those planned by ESO of $42 \mathrm{~m}$ aperture diameter. Therefore, to determine the dimension of the laser sources, the $\mathrm{AO}$ systems and guarantee high reliability in terms of useful nights, we are interested in studying the sodium layer column abundance, the centroid height, the sodium concentration, and the layer thickness. The layer thickness is related to the LGS elongation observed at the edges of an extremely large telescope. To estimate all of these parameters, a rich collection of experimental data accumulated 
during a long period of measurements (1973-2008) is analyzed in this paper.

\section{Experiment}

The Brazilian National Space Research Institute (Instituto National de Pesquisas Espaciais-INPE-MCT) conducts experimental measurements of the mesospheric sodium layer characteristics in São José dos Compos, São Paulo, at a latitude $23^{\circ}$ south. The Lidar (Light Detection And Ranging) technique was used to measure the evolution of the mesospheric sodium layer over a long period (1973-2008). A description of the measurement technique and discussion of the measurement precision can be found in Simonich et al. (1979) and in Clemesha et al. (1992). The data used in this study consists of the height of the maximum sodium concentration, the centroid height, the full width at half maximum (FWHM) of the equivalent Gaussian of the mesospheric sodium layer thickness, the peak of the sodium concentration, and the column abundance. The Lidar signal is measured using a photon-counting technique. For various reasons (including, of course, poor seeing) the photon counts are often low and the noise level high. The poorest quality data were removed from the analyzed data.

The early data (up to 1980) were taken at height intervals, so the height of peak sodium concentration was quantized into $2 \mathrm{~km}$ intervals. The centroids from this data are, however, good to a fraction of a $\mathrm{km}$. Sporadic sodium layers, frequently observed, can be a few km thick with peak concentrations as high as 10 times the normal mesospheric sodium density. These sporadic layers have a strong effect on the layer statistics.

The absolute Na concentration for the periods (1973-1980) and (2007-2008) is more accurately calibrated than for the data (1980-2005). Depending on the photon counts, the accuracy of the centroid height measurements can be approximately $\pm 10 \mathrm{~m}$. Heights are measured relatively to the Lidar station altitude, which is $625 \mathrm{~m}$ above sea level.

\section{Experimental setup}

The Fig. 1 shows a simplified diagram of the INPE Lidar in its present configuration. A description of the Lidar can be found in Clemesha et al. (2001) and in Simonich et al. (1979). Over the many years during which the system has operated, it has undergone considerable modifications, and only the $75 \mathrm{~cm}$ receiving telescope and the $120 \mathrm{~cm}$ turning mirror have remained unchanged. Currently the $589.0 \mathrm{~nm}$ laser pulse needed to probe the atmospheric sodium $D_{2}$ line is generated by mixing the 1064 and $1319 \mathrm{~nm}$ outputs from seeded neodymium Yag lasers. This produces approximately $10 \mathrm{~ns} 50 \mathrm{~mW}$ pulses at $10 \mathrm{pps}$ with a bandwidth of about $100 \mathrm{MHz}$. By thermally tuning the $1064 \mathrm{~nm}$ seeder laser, it is possible to tune the $589.0 \mathrm{~nm}$ output and investigate the fine structure of the $D_{2 a}$ line, making it possible to determine the Doppler temperature of the atmospheric Na atoms. All the data analyzed in this paper were taken by using either a wide-band laser or the laser tuned to the $D_{2 a}$ peak. The laser bandwidth was taken into account when determining the absolute atmospheric sodium concentrations. In the early data, this involved periodically measuring the laser bandwidth using a Fabry-Perot interferometer, and monitoring the behavior of the laser during data acquisition, by sampling its output with the aid of a sodium vapor cell. For the narrow-band laser described above, the output was tuned to either the $D_{2 a}$ peak or the crossover minimum between $D_{2 a}$ and $D_{2 b}$. In the present analysis, only $D_{2 a}$ peak data were used.

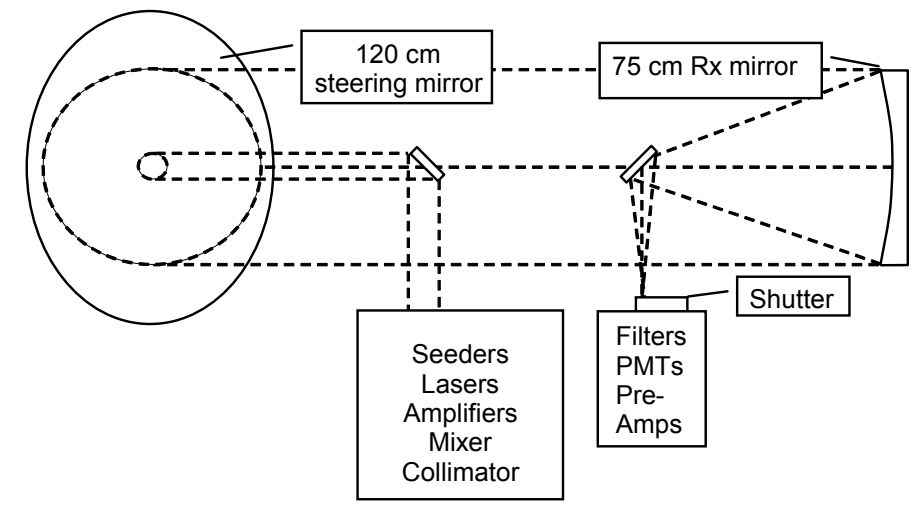

Fig. 1. Simplified plan view: optical layout for INPE Lidar.

The Lidar receiver uses a $75 \mathrm{~cm}$ diameter primary mirror to concentrate the received signal onto two thermoelectrically cooled photomultipliers used in photon-counting mode. We note that $95 \%$ of the received signal goes to one pmt and $5 \%$ to the other, thus extending the dynamic range of the receiver. This is necessary because the same Lidar is used to study stratospheric aerosols at around $20 \mathrm{~km}$, the return from which is much stronger than from the mesosphere. A rapidly rotating mechanical shutter, synchronized to the laser pulse, protects the pmts from the strong signal scattered from the troposphere, and sky noise is minimized using narrow-band interference filters.

The received signal is registered as photon counts in multichannel scalers. In the early days of the Lidar, a scaler channel width of $13.3 \mu$ s was used, corresponding to a height resolution of $2 \mathrm{~km}$. More recent data used channel widths corresponding to either $300 \mathrm{~m}$ or $250 \mathrm{~m}$. Different time resolutions (given by the summation time of the multi-channel scaler) have been used at different times, typically between 1 and 5 min. Most of our measurements were performed at night to avoid the strong noise source constituted by the sunlit sky, although some daytime data were acquired for studies of the diurnal variation in atmospheric sodium. Depending on weather conditions and data rates, a maximum of about 600 independent profiles are obtained in one night's data.

Absolute calibration of a Lidar is a difficult task, partly because it is not easy to accurately measure the system efficiency but, more importantly, because there is considerable uncertainty in the transmission of the lower atmosphere. This problem is avoided in sodium measurements by comparing the sodium signal with that from the upper stratosphere, around $40 \mathrm{~km}$, where the atmospheric density is accurately known and aerosols are rare. Absolute calibration then only involves knowledge of the relative photon counts and scattering cross-sections (resonant for the sodium and Rayleigh for the stratosphere).

\section{Data presentation}

We present in Figs. 2-4, an illustration of the experimental measurement distribution of three parameters of the mesospheric sodium layer: the column abundance, the centroid height, and the sodium layer thickness. We should mention that the data analysis covers mainly three time periods. The number of the sodium vertical distribution profiles measured for each period is 58625 for the period (1973-2008), 10780 for the period (1973-1980), and 24969 for the period (2007-2008).

The Fig. 5 presents the monthly mean values of the column abundance, centroid height, sodium layer thickness, and 


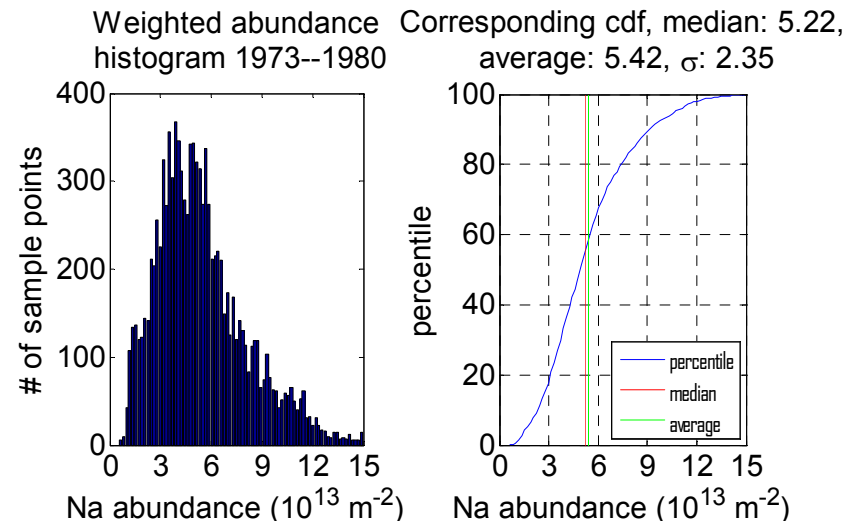

Fig. 2. Weighted histogram and the cumulative distribution function of column abundance (1973-1980).

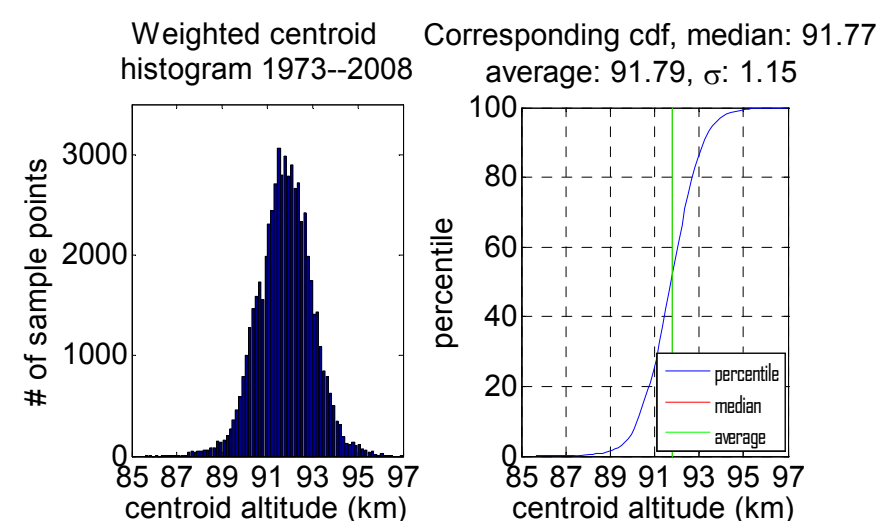

Fig. 3. Weighted histogram and the cumulative distribution function of the sodium layer centroid height.
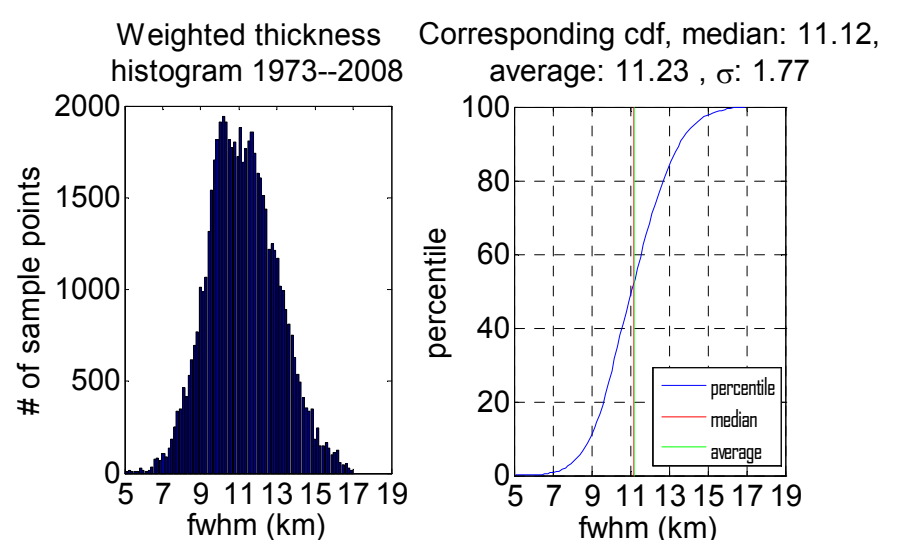

Fig. 4. Weighted histogram and the cumulative distribution function of sodium layer thickness. Note that the average layer thickness is larger than $10 \mathrm{~km}$, the value used in most published AO simulations.

height of the maximum sodium concentration as functions of time. Accurate measurements of the laser bandwidth were not made between 1981 and 2006, so data from this period were not included in the abundance analysis (see Fig. 5a). The median of the column abundance is $5 \times 10^{13} \mathrm{~m}^{-2}$. We observe that the column abundance can reach maximum values of $16 \times 10^{13} \mathrm{~m}^{-2}$, which may be caused by sporadic layers.

The altitude variation in the sodium layer centroid is a very important phenomenon to consider because in AO closed loop operation it can introduce an offset error in the focus compensation.The mean centroid height is found to vary between $90 \mathrm{~km}$
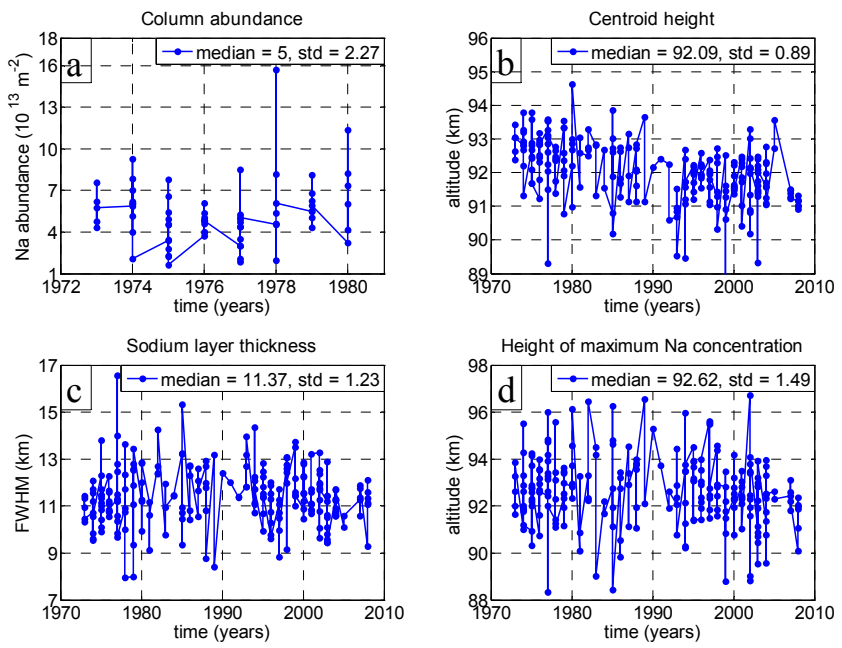

Fig. 5. Time series of mesospheric sodium layer characteristics. Solid dots represent monthly mean values of each parameter.

and $94 \mathrm{~km}$ and its median is $92.09 \mathrm{~km}$, as seen in Fig. 5b. The sodium layer thickness obtained from its equivalent Gaussian shown in Fig. $5 \mathrm{c}$ varies between $8 \mathrm{~km}$ and $15 \mathrm{~km}$. Its median is $11.37 \mathrm{~km}$. The height of maximum sodium concentration varies between $90 \mathrm{~km}$ and $97 \mathrm{~km}$ with a median of $92.62 \mathrm{~km}$. We observe that the height of the maximum sodium concentration is generally larger than the centroid height. Chemistry plays a key role in the equilibrium between the atomic $\mathrm{Na}$ and other atoms, ions, and molecules in the upper atmosphere, as described in the following section.

\section{Mesospheric sodium layer chemistry}

Sodium ions $\left(\mathrm{Na}^{+}\right)$and sodium bicarbonate $\left(\mathrm{NaHCO}_{3}\right)$ are the main reservoir species above and below the atomic mesospheric sodium layer, respectively (Fan et al. 2007; Plane et al. 1999). Because of the long residence time ( $\sim 7$ days) of ablated sodium in the region between 80 and $100 \mathrm{~km}$, and the comparatively short lifetimes of the rate-determining chemical reactions that convert sodium between atomic $\mathrm{Na}$ and these reservoirs, the chemistry reaches a photochemical steady-state on the timescale of vertical transport by eddy or molecular diffusion. The chemical reactions responsible for the conversion of $\mathrm{Na}$ to $\mathrm{NaHCO}_{3}$ occur rapidly and have small temperature dependencies (Plane 2004, 1998). $\mathrm{NaHCO}_{3}$ is converted back to $\mathrm{Na}$ by the reaction with $\mathrm{H}$. This reaction has a large activation energy, that is at higher temperatures it becomes much faster, and the steady-state balance shifts from $\mathrm{NaHCO}_{3}$ to $\mathrm{Na}$ (Cox et al. 2001). It was suggested that the final sink for sodium on the bottomside of the layer is attachment to aerosol particles (Hunten et al. 1980).

\section{Sodium layer parameters variation}

We now focus on the variation in the column abundance, the centroid height, and the sodium layer thickness, because these characteristics are very important for the estimation of the laser guide star parameters (sodium photon return, spot size, and spot elongation). The daily medians of the sodium layer parameters calculated over all the experimental period (1973-2008) are presented in Fig. 6. 


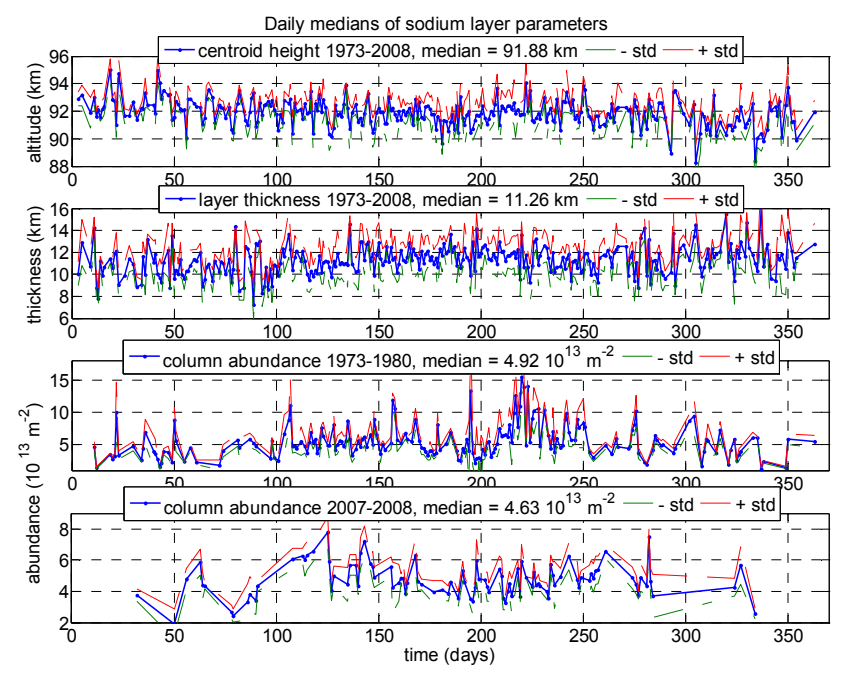

Fig. 6. Daily medians of sodium layer parameters, where the dashed lines represent the standard deviations for all the data each day.

The sodium thickness $t_{\mathrm{Na}}$ can be defined for arbitrary sodium profiles, $\chi_{\mathrm{Na}}$, as

$t_{\mathrm{Na}}=\frac{b}{C_{\mathrm{Na}}} \int_{\text {atmo }} \mid H-H_{\mathrm{Na}} \chi_{\mathrm{Na}}(H) \mathrm{d} H$.

The centroid height, $H_{\mathrm{Na}}$, is defined as

$H_{\mathrm{Na}}=\frac{\int_{\mathrm{atmo}} H \chi_{\mathrm{Na}}(H) \mathrm{d} H}{C_{\mathrm{Na}}}$.

The sodium column density $C_{\mathrm{Na}}$ is defined as

$C_{\mathrm{Na}}=\int_{\text {atmo }} \chi_{\mathrm{Na}}(H) \mathrm{d} H$

where $b=2(\ln 2)^{1 / 2}, H$ is the altitude above sea level, $\chi_{\mathrm{Na}}$ is the sodium density per unit volume (unitatoms $/ \mathrm{m}^{3}$ ), and the integration is performed vertically through the entire atmosphere. With this definition, $t_{\mathrm{Na}}$ equals the FWHM thickness of the sodium layer if $\chi_{\mathrm{Na}}(\mathrm{H})$ has a Gaussian shape

$\chi_{\mathrm{Na}}(H)=\frac{b}{\sqrt{\pi}} \frac{C_{\mathrm{Na}}}{t_{\mathrm{Na}}} \exp \left(-\left(b \frac{H-H_{\mathrm{Na}}}{t_{\mathrm{Na}}}\right)^{2}\right)$,

hence $\chi_{\mathrm{Na}}\left(H_{\mathrm{Na}} \pm t_{\mathrm{Na}} / 2\right) / \chi_{\mathrm{Na}}\left(H_{\mathrm{Na}}\right)=1 / 2$. We present the mesospheric sodium layer parameters whose variations are important for astronomical observations. We calculated the daily median value for each parameter for all the experimental period (1973-2008). Each daily median represents the median for the same day of the year for all the experimental period, around 100 experimental measurements for each median value. The experimental times are randomly chosen, so the statistical accuracy of our calculation should be very high. Figure 6 represents three important characteristics, of the mesospheric sodium layer, for the laser guide star adaptive optics system. From top to bottom, it displays the daily medians of: the centroid height, the sodium layer thickness, and the sodium column abundance. The evolution of each parameter is represented by a solid blue line. The standard deviation of each daily median is represented by dashed lines (up + std in red, down - std in green).

The annual median of the centroid height is $91.88 \mathrm{~km}$. The daily median of this height varies between a maximum of $95 \mathrm{~km}$

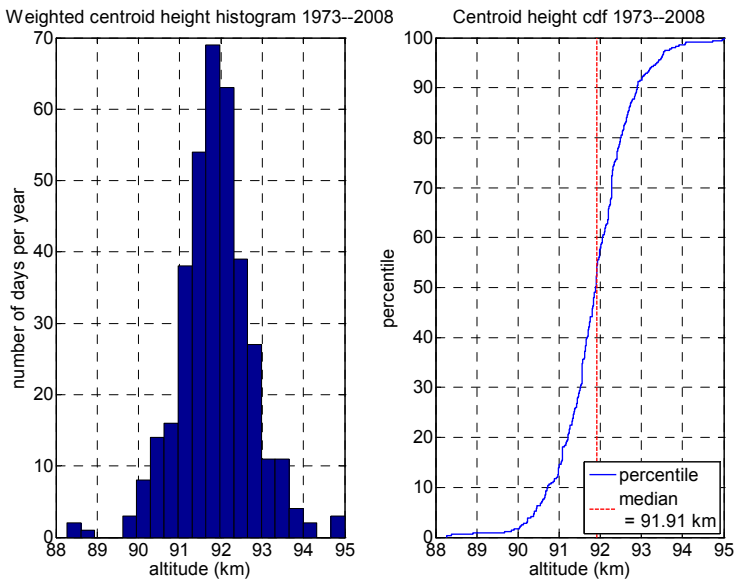

Fig. 7. Weighted histogram and the cumulative distribution function of centroid height.

and a minimum of $88 \mathrm{~km}$. The layer thickness is calculated from its equivalent Gaussian. The complexity of the mesospheric sodium layer structure means that the Gaussian fits are not always very meaningful, although this information could be very useful for the determination of the LGS spot elongation. The annual median of the full width at half maximum (FWHM) of the sodium layer is $11.26 \mathrm{~km}$. The daily median of this thickness varies between a maximum of $16 \mathrm{~km}$ and a minimum of $8 \mathrm{~km}$. The annual median of the sodium column abundance is $4.92 \times 10^{13} \mathrm{~m}^{-2}$ for the period (1973-1980) and $4.63 \times 10^{13} \mathrm{~m}^{-2}$ for the period (2007-2008). Its daily median sometimes reaches a maximum of $20 \times 10^{13} \mathrm{~m}^{-2}$. This high value could be explained by the presence of sporadic layers, although the presence of these layers does not appear to significantly affect long-term averages (Simonich et al. 2005).

Figures 7-9 present a statistical calculation of the mesospheric sodium layer parameters for the data of the full observing period, without daily averages. Figure 7 shows that the centroid height is more than $92 \mathrm{~km}$ during $40 \%$ of the year, with a median value of $91.91 \mathrm{~km}$. The velocity of vertical displacement of the centroid height is an important parameter in designing a LGS system. Figure 8 shows that the centroid vertical velocity varies between 0 and $5 \mathrm{~m} / \mathrm{s}$, with a median value of $0.52 \mathrm{~m} / \mathrm{s}$. During $60 \%$ of the year, this velocity is less than $0.5 \mathrm{~m} / \mathrm{s}$. Figure 9 shows that the mesospheric sodium layer thickness is between $7 \mathrm{~km}$ and $16 \mathrm{~km}$, its median value being $11.31 \mathrm{~km}$, which is less than its median value during $60 \%$ of the year.

For the column abundance, because of the bandwidth calibration problem mentioned in Sect. 2, we considered two periods (1973-1980) and (2007-2008). The close agreement between the median abundances $4.92 \times 10^{13} \mathrm{~m}^{-2}$ for the first period and $4.63 \times 10^{13} \mathrm{~m}^{-2}$ for the second (see Fig. 6) suggests that the data for the two periods could be combined to improve the statistics, as shown in Fig. 10.

\subsection{Estimation of the return flux availability}

To estimate the required laser power for the use of adaptive optics, we calculate the specific return photon flux to the ground. The expression of this parameter is given by

$F_{R}(\zeta)=\frac{s_{\mathrm{ce}} C_{\mathrm{Na}} T_{\mathrm{atmo}}^{2 \sec (\zeta)}}{H_{\mathrm{Na}}^{2} \sec (\zeta)}$ 

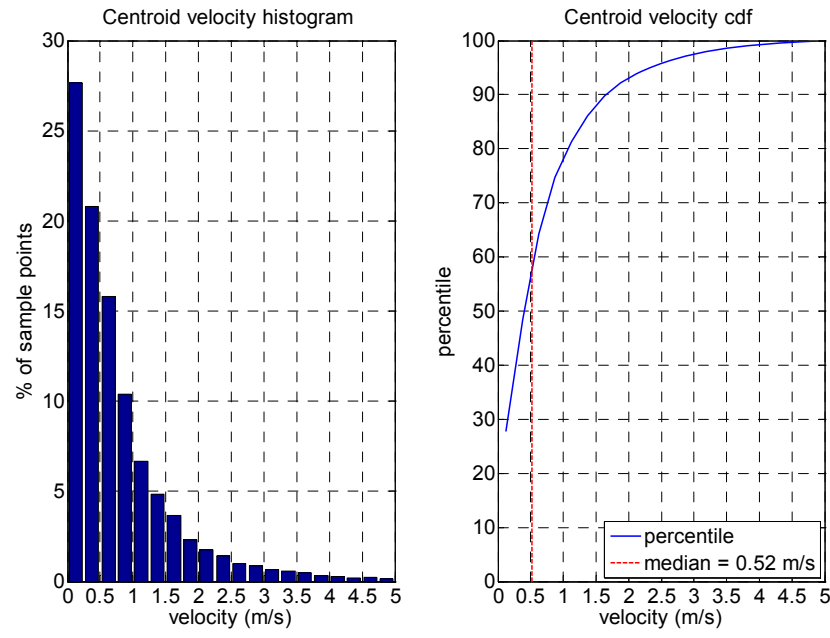

Fig. 8. Histogram and the cumulative distribution function of sodium layer centroid velocity, for all the experimental period (1973-2008).
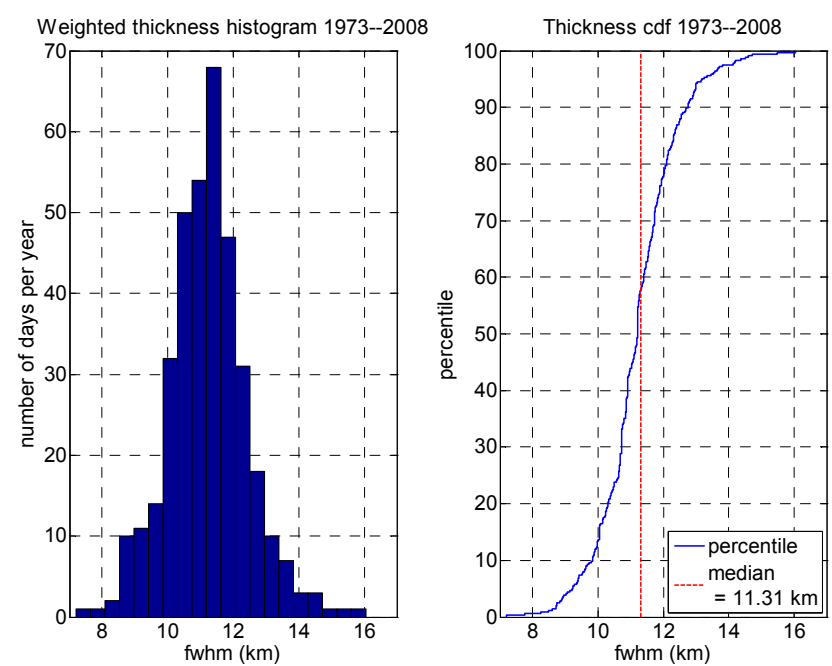

Fig. 9. Weighted histogram and the cumulative distribution function of sodium layer thickness.

where $F_{R}$ is the specific return photon flux to the ground per watt of launched laser power, where $s_{\mathrm{ce}}$ is the coupling efficiency of light at $589 \mathrm{~nm}$ to the sodium atoms (unitphotons $\times$ $\mathrm{m}^{2} / \mathrm{s} / \mathrm{W} /$ atom), $C_{\mathrm{Na}}$ is the vertical column density of sodium, $\sec (\zeta)$ is the secant of the zenith angle $\zeta$ (the airmass), $T_{\text {atmo }}$ is the single-pass transmission of the atmosphere at $589 \mathrm{~nm}$ at zenith, and $H_{\mathrm{Na}}$ is the vertical distance from the telescope to the sodium layer centroid. We note that $s_{\mathrm{ce}}$ and thus $F_{R}$ strongly (and to some extent nonlinearly) depend on laser power, laser spectrum, laser polarization, and angle between laser propagation direction and the magnetic field (Moussaoui et al. 2008, 2009), in addition to pulse format if any.

For vertical propagation of the laser beam and assuming that the centroid height is constant and the coupling efficiency of light at the sodium wavelength is constant, the return flux can be assumed to be proportional to the sodium column density. Assuming that a laser power of $100 \%$ is needed to produce sufficent sodium laser-guide-star photon return-flux for a median value of a column abundance of $4 \times 10^{13} \mathrm{~m}^{-2}$, we calculate the number of days for which the sodium laser-guide-star return is enough for the adaptive optics (AO) system for low geographic latitude sites as shown in Fig. 11.
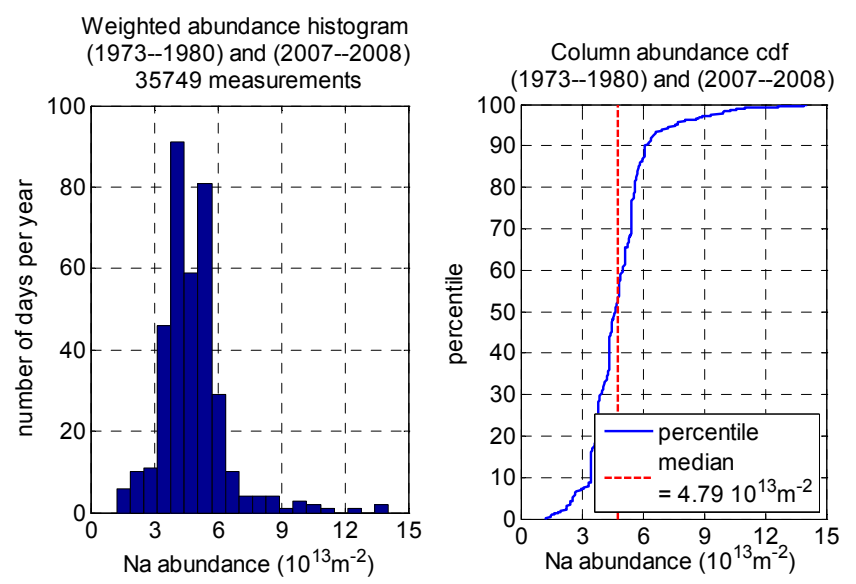

Fig. 10. Weighted histogram and the cumulative distribution function of column abundance for the combined periods (1973-1980) and (2007-2008).

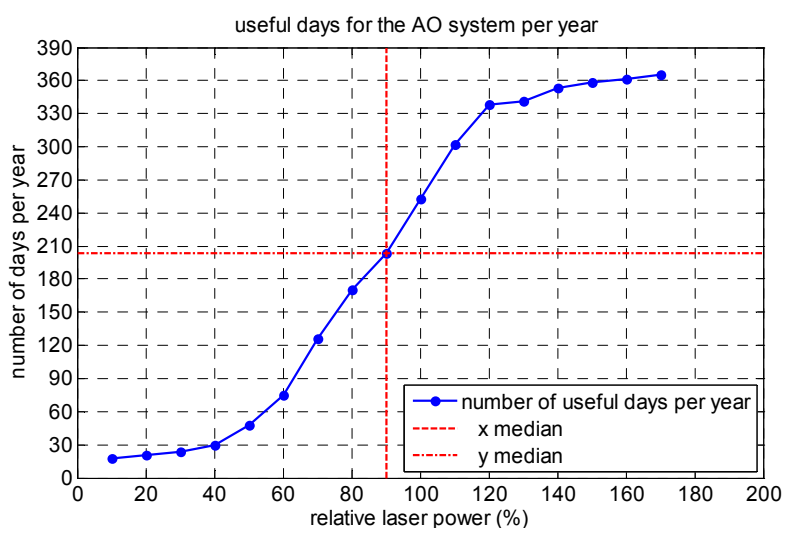

Fig. 11. Number of useful days per year as function of the Laser power.

From Fig. 11, we can see that for a laser power of $100 \%$, the return flux is assumed to be enough for the $\mathrm{AO}$ system during 253 days per year. If we increase the laser power by a factor of $20 \%$ the number of useful days for the AO system is around 338 days per year. So the observation with an adaptive optics system using laser guide stars can practically cover the whole year for a relative laser power of $120 \%$.

\subsection{The LGS spot elongation}

The laser-guide-star photon return flux and hence the power requirement are driven by the spot elongation. It is useful to know the evolution of this parameter, to optimize the characteristics of the sodium laser guide star. The angular spot elongation observed from the ground (see Fig. 12) is given by

$\varepsilon=\frac{t_{\mathrm{Na}} R}{\sec (\zeta) H_{\mathrm{Na}}^{2}}$,

where $t_{\mathrm{Na}}$ is the vertical FWHM sodium layer thickness and $R$ is the orthogonal offset of the observer from the laser beam.

Figure 13 presents a statistical calculation of the quantity $\left(t_{\mathrm{Na}}\right) /\left(H_{\mathrm{Na}}\right)^{2}$. For a given zenith angle $\zeta$ and an orthogonal offset $R$, this quantity determines the spot elongation. Figure 14 presents a quantitative estimation of the spot elongation of the sodium laser guide star in arcsec, for a vertical propagation of the laser beam and an orthogonal offset $R=21 \mathrm{~m}$, which corresponds to the radius of the aperture of the European Extremely 


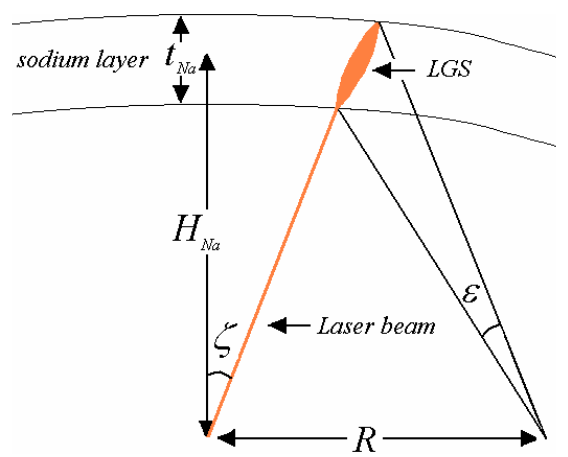

Fig. 12. Schematic drawing of the angular spot elongation as observed from the ground.
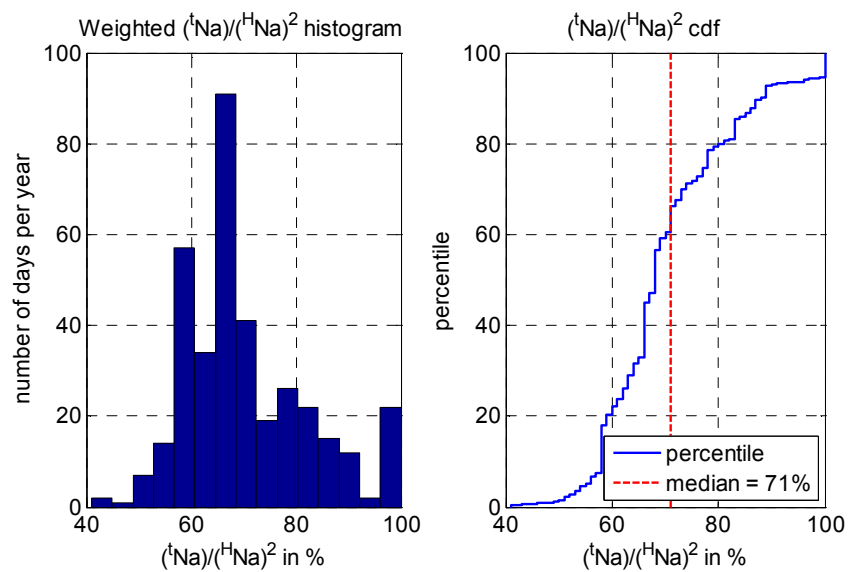

Fig. 13. Weighted histogram and the cumulative distribution function of the ratio of the layer thickness to the squared centroid height. The latter is a measure of the spot elongation for all the experimental period (1973-2008).

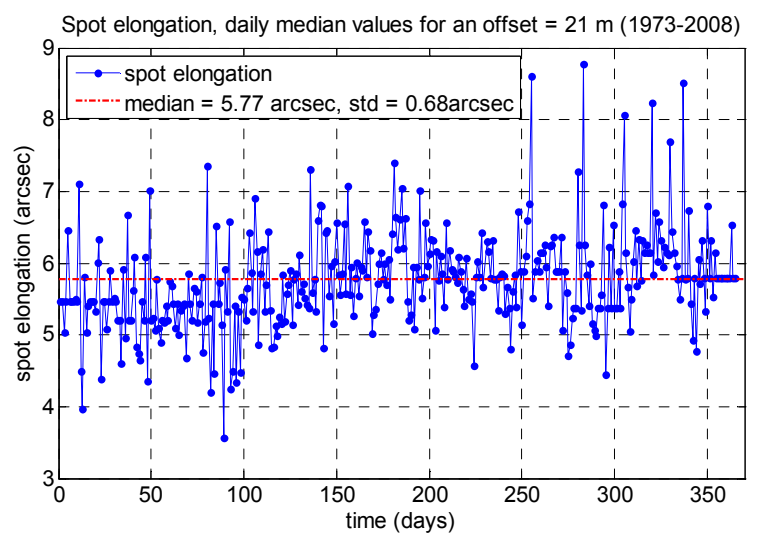

Fig. 14. Spot elongation for a vertical propagation of the laser beam and an offset distance of $R=21 \mathrm{~m}$.

Large Telescopes (E-ELT) planned by ESO (with $42 \mathrm{~m}$ aperture diameter).

The values of the sodium layer thickness and the centroid height are the daily medians calculated over all the period (1973-2008). We can see that the spot elongation for an offset distance of $R=21 \mathrm{~m}$, is situated between 3 and 9 arcsec, and that its daily median is 5.77 arcsec with a standard deviation of 0.68 arcsec. If we consider the maximum value of 9 arcsec in Fig. 14, we see from Fig. 13 that the spot elongation is less than 6.4 arcsec for more than $60 \%$ of the year.
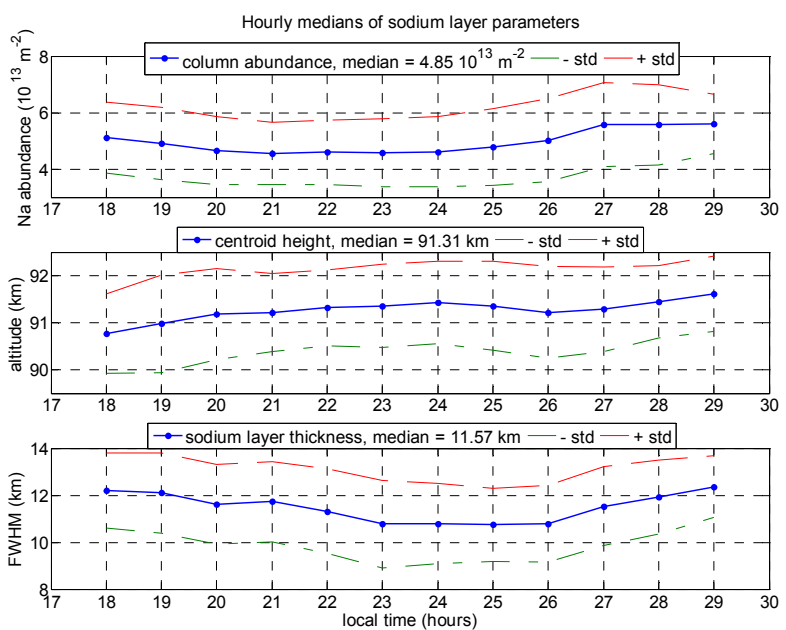

Fig. 15. Hourly medians of sodium layer parameters, the dashed curves represent the standard deviations for all the data for each hour. Time $=30 \mathrm{~h}$ means 0600 local time.

\section{Nocturnal variation in the sodium layer parameters}

Figure 15 shows the hourly median values of the centroid height, the thickness, and the column abundance of the sodium layer. The derivation of a median nocturnal variation is complicated because most of the experimental observations refer to time periods of only a few hours for a given night. As a result of this, the very large night-to-night variations that frequently occur will tend to make the mean nocturnal variation noisier if any simple median is taken. To overcome this problem, we selected nights with enough data to cover many hours of the same night. The hourly median values presented in Fig. 15 are calculated for about 25000 measurements.

The column abundance varies between a minimum of $4.5 \times$ $10^{13} \mathrm{~m}^{-2}$ just before midnight and reaches its maximum value of more than $5.5 \times 10^{13} \mathrm{~m}^{-2}$ at 0500 local time $(29 \mathrm{~h}$ means five hours after midnight). Its nocturnal median is $4.85 \times 10^{13} \mathrm{~m}^{-2}$. The centroid height increases during the night, and reaches a maximum of $91.50 \mathrm{~km}$ at 0500 local time. Its nocturnal median is $91.31 \mathrm{~km}$. The sodium layer thickness starts the night by decreasing until it reaches a minimum of $10.5 \mathrm{~km}$ around midnight and increases after that to reach a maximum around $12 \mathrm{~km}$ at 0500 local time. Its nocturnal median is $11.57 \mathrm{~km}$. It should be noted that these nocturnal variations are almost certainly tidal in origin (Batista et al. 1985) and since the phase and amplitude of atmospheric tides vary during the year (with a mainly semi-annual oscillation at low latitudes), the temporal variations shown in Fig. 15 are useful only when considered as annual averages.

\section{Long term evolution of the sodium layer parameters}

\subsection{Column abundance}

In Fig. 16, we present monthly medians of the sodium column abundance with circular dots and a solid blue line. The dashed red and green lines represent respectively \pm standard deviation from the monthly median of the column abundance calculated over the entire experimental period.

Figure 16 shows the values of the monthly medians of the column abundance. Each monthly median corresponds to the 


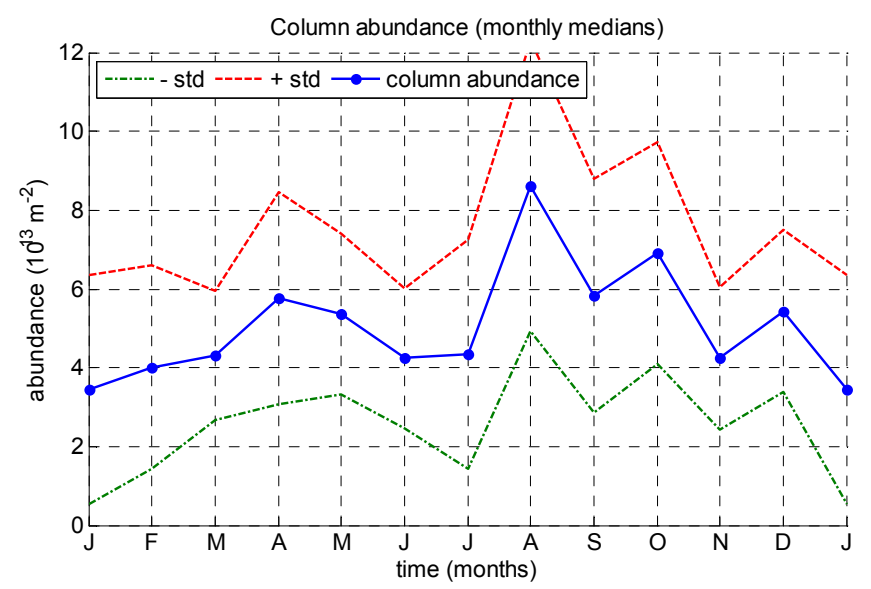

Fig. 16. Monthly medians of the mesospheric sodium column abundance, where the standard deviations are calculated for each month throughout the experimental period.

median of the accumulated data for the same month over the entire experimental measurement period. The sodium column abundance reaches a maximum during the period of (JulyAugust), and a minimum around (February-January). Several authors, such Cox et al. (2001), Xiong et al. (2003), and Drummond et al. (2007) noted that the time evolution of the mesospheric sodium column abundance has a characteristic period of one year. It is tempting to suggest that the maximum of the sodium column abundance could be explained by the increase in the meteoric input during this period, which is rich in terms of meteor showers. The most visible meteor shower called the Perseids occurs each year during the period (July 17 to August 24), and peaks in mid-August at over 1 meteor a minute. Another strong meteor shower is observed during this period called south Delta Aquarids (July 12 to August 19). However, it has long been known that the sodium layer variation is seasonal, rather than annual, with a winter abundance maximum in both the northern and southern hemispheres. This winter maximum appears to be the effect of the seasonal temperature variation in the reactions that recycle sodium compounds back to free sodium (Plane et al. 1999).

\subsection{Centroid height}

To determine in more detail the centroid height evolution during long-term observations we calculated its monthly medians. Each median was calculated for a given month of the year on the basis of the entire period of the data measurements. In Fig. 17, these values are represented by circular dots and a solid blue line. The dashed red and green lines represent respectively \pm the standard deviation of the monthly medians of the centroid height calculated over the entire experimental period.

The sharp minimum in November appears to represent a true geophysical effect which is believed to be tidal in origin (Clemesha et al. 2005). Apart from this November minimum, the centroid height appears to exhibit a predominantly semi-annual variation, in agreement with Xiong et al. (2003). A semi-annual oscillation is likely to be associated with tides and gravity waves (Clemesha et al. 2005, 2009). It is possible that the August maximum in centroid height is related to the strong meteor showers that occur in that month.

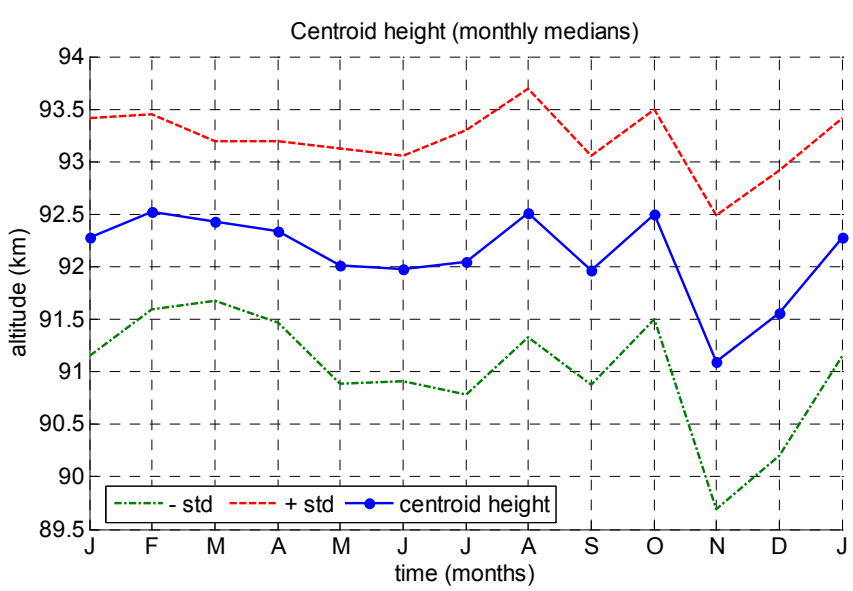

Fig. 17. Monthly medians of the mesospheric sodium layer centroid height, the standard deviations are calculated for each month throughout the experimental period.

\section{Conclusion}

This paper has analyzed a large sample of mesospheric sodium data, collected in the southern hemisphere over 35 years, at latitudes similar to that of the ESO Paranal observatory in Chile, to derive statistically meaningful parameters relevant for adaptive optics with laser guide stars at $589 \mathrm{~nm}$. This analysis has provided much needed mesospheric sodium parameter statistics, which are meaningful because they were obtained for more than a few samples.

The knowledge of the statistics of the mesospheric sodium layer parameters allows those developing adaptive optics with sodium LGSs to better simulate and design the LGS-AO systems more accurately, as well as to dimension the laser system to secure the availability for observations. This knowledge is in particularly important for the design e.g, of the second generation ESO VLT and the new E-ELT LGS-AO systems.

Assuming that for a given laser power and a sodium column abundance of $4 \times 10^{13} \mathrm{~m}^{-2}$, the photon return flux is sufficient for the $\mathrm{AO}$ system, the telescope can observe with the $\mathrm{AO}$ system using sodium LGSs, over more than 250 days per year. An increasing of the laser power by $20 \%$ allows us to cover the entire year.

The specific photon return flux reaches its maximum values during July-August in the southern hemisphere. This effect can be explained by the increase in meteoric ablation during this period, which is rich in terms of meteoric showers, and/or by the mesospheric sodium chemistry, which is related to the winter maximum in mesopause region temperature.

The median value of the sodium LGS spot elongation for an offset distance of $R=21 \mathrm{~m}$, is $5.77 \mathrm{arcsec}$. The average values of the sodium layer parameters calculated over all the experimental period (1973-2008) are $92.09 \mathrm{~km}$ for the centroid height, $11.37 \mathrm{~km}$ for the layer thickness, and $5 \times 10^{13} \mathrm{~m}^{-2}$ for the column abundance. The experimental observation times are distributed randomly, and 58625 sodium profiles data have been taken into account, so the statistical accuracies of our measurements should be high.

Acknowledgements. This work was possible thanks to the R\&D activities of the Laser Systems Department, funded by the ESO Technology Division under Job994. For the Lidar measurements, financial support has been received from the Fundação de Amparo à Pesquisa do Estado de São Paulo (FAPESP) and the Conselho Nacional de Desenvolvimento Cientifico e Tecnológico (CNPq). 


\section{References}

Batista, P. P., Clemesha, B. R., Simonich, D. M., \& Kirchhoff, V. W. J. H. 1985, J. Geophys. Res., 90, 3881

Clemesha, B. R., Kirchhoff, V. W. J. H., \& Simonich, D. M. 1979, Planet. Space Sci., 27, 909

Clemesha, B. R., Simonich, D. M., \& Batista, P. P. 1992, Geophys. Res. Lett., 19,457

Clemesha, B. R., Batista, P. P., \& Simonich, D. M. 2001, Adv. Space Res., 27, 1679

Clemesha, B. R., Simonich, D. M., Vondrak, T.,\& Plane, J. M. C. 2004, J. Geophys. Res., 109, D05302

Clemesha, B. R., Simonich, D. M., Batista, P. P., \& Takahashi, H. 2005, Adv. Space Res., 35(11), 1951

Clemesha, B. R., Batista, P. P, Costa, R. B. D., \& Schuch, N. 2009, Annales Geophysicae, 27, 1059

Cox, R. M., Self, D. E., \& Plane, J. M. C. 2001, J. Geophys. Res., 106, 1733

Drummond, J., Novotny, S., Denman, C., et al. 2007, The Sodium LGS Brightness Model over the SOR, AMOS Technical Conference

Fan, Z. Y., Plane, J. M. C., Gumbel, J., Stegman, J., \& Llewellyn, E. J. 2007, Atmos. Chem. Phys., 7, 4107

Gardner, C. S., Senft, D. C., \& Kwon, K. H. 1988, Nature, 332, 142
Gardner, C. S., Plane, J. M. C., Pan, W., et al. 2005, J. Geophys. Res., 110, D10302

Hunten, D. M., Turco, R. P., \& Toon, O. B. 1980, J. Atmos. Terr., 37, 1342

Kirchhoff, V. W. J. H., \& Clemesha, B. R. 1983, J. Geophys. Res., 88, 442 Megie, G., \& Blamont, J. E. 1977, Planet. Space Sci., 25, 1093

Moussaoui, N., Holzlöhner, R., Hackenberg, W., \& Bonaccini Calia, D. 2008, Proc. SPIE, 7015, 70152W1-10

Moussaoui, N., Holzlöhner, R., Hackenberg, W., \& Bonaccini Calia, D. 2009, A\&A, 501, 793

Plane, J. M. C. 2003, Chem. Rev., 103, 4963

Plane, J. M. C. 2004, Atmos. Chem. Phys., 4, 627

Plane, J. M. C., Cox, R. M., Qian, J., et al. 1998, J. Geophys. Res., 103, 6381

Plane, J. M. C., Gardner, C. S., Yu, J. R., et al. 1999, J. Geophys. Res., 104, 3773

She, C. Y., Chen, S. S., Hu, Z. L., et al. 2000, Geophys. Res. Lett., 27, 3289

Simonich, D. M., Clemesha, B. R., \& Kirchhoff, V. W. J. H. 1978, Trans. Am. Geophys. Union, 59, 342

Simonich, D. M., Clemesha, B. R., \& Kirchhoff, V. W. J. H. 1979, J. Geophys. Res., 84, 1543

Simonich, D. M., Clemesha, B. R., \& Batista, P. P. 2005, Adv. Space Sci., 35(11), 1976

Tilgner, C., \& von Zahn, U. 1988, J. Geophys. Res., 93, 8439

Xiong, H. U., Gardner, C. S., \& Liu, A. Z. 2003, Chin. J. Geophys., 46, 3, 432

von Zahn, U., Hansen, G., Kurzawa, H., et al. 1988, Nature, 331, 594 